\title{
Surveillance of mother to child transmission of HIV in Romania, a 12 years' experience in the National Institute for Infectious Diseases "Prof. Dr. Matei Balş"
}

\author{
Mariana Mărdărescu', Cristina Petre ${ }^{1 *}$, Adrian Streinu-Cercel ${ }^{1,2}$, Sorin Petrea', Ruxandra Neagu-Drăghicenoiu', \\ Rodica Ungurianu', Ana Maria Tudor ${ }^{1,2}$, Alina Cibea', Delia Vlad', Mihai Mitran³, Otilia Benea ${ }^{1,2}$, Dan Oțelea?', \\ Carmen Crăciun', Tatiana Colțan', Marieta lancu', lonel lonel ${ }^{4}$, Alexandra Mărdărescu ${ }^{5}$
}

From The 9th Edition of the Scientific Days of the National Institute for Infectious Diseases Prof Dr Matei Bals Bucharest, Romania. 23-25 October 2013

\section{Background}

The latest WHO data regarding HIV/AIDS infection have revealed the real dimension of this epidemic. In Romania, in 2012, out of the 754 new detected cases, 19 represented children between $0-14$ years. Vertical transmission was recorded for 16 (2.6\%) of these cases. Within this epidemiological context, mother to child transmission continues to represent a top theme, which determined us to continue with the observations initiated in 2000 on this phenomenon.

\section{Methods}

During 01 January 2000 - 31 December 2012, children from all around the country were included in the study and assessed at the National Institute for Infectious Diseases "Prof. Dr. Matei Balş" in Bucharest. They were surveyed clinically and biologically until the age of 18 months. Relevant data on the children were recorded: gender, age, time of diagnosis, antiretroviral therapy (ART) prophylaxis (YES or NO), type of birth and nourishment, CD4 count, viral load (VL) at baseline and at the end of the surveillance period. When assessing the mothers we focused on: age, environment, level of education, occupation, time of HIV infection diagnosis, treatment/prophylaxis, type of birth, CD4 and VL at birth (data obtained from medical records or anamnesis).

\footnotetext{
* Correspondence: croxpetre@yahoo.com

${ }^{1}$ National Institute for Infectious Diseases "Prof. Dr. Matei Balş", Bucharest, Romania

Full list of author information is available at the end of the article
}

\section{Results}

We surveyed 517 children with ages between 0-18 months. Of these children under observation for 12 years, 15\% were considered infected with HIV. This rate decreased in stages, from $45 \%$ in 2005 to $17 \%$ in 2010 . The main transmission causes were late HIV infection diagnosis of the mother, lack of prophylaxis/treatment for mothers, vaginal birth, breastfeeding, lack of prophylaxis in children or tardiness in initiating it.

\section{Conclusion}

Despite active measures, the rate of HIV transmission remains very high. The elimination of vertical transmission is considered a realistic objective of public health policies. The collaboration and active involvement of specialists from various medical and social domains is a necessity.

\section{Authors' details}

1National Institute for Infectious Diseases "Prof. Dr. Matei Balş", Bucharest, Romania. ${ }^{2}$ Carol Davila University of Medicine and Pharmacy, Bucharest, Romania. ${ }^{3}$ Clinical Hospital of Obstetrics and Gynecology "Prof. Dr. Panait Sârbu", Bucharest, Romania. ${ }^{4}$ National Centre for Transmissible Diseases Surveillance and Control (CNSCBT), Bucharest, Romania. ${ }^{5}$ Romanian HIV/AIDS Centre, National Institute for Infectious Diseases "Prof. Dr. Matei Balş", Bucharest, Romania.

Published: 16 December 2013

doi:10.1186/1471-2334-13-S1-01

Cite this article as: Mărdărescu et al.: Surveillance of mother to child transmission of HIV in Romania, a 12 years' experience in the National Institute for Infectious Diseases "Prof. Dr. Matei Balş". BMC Infectious Diseases 2013 13(Suppl 1):01

\section{C) Biomed Central}

๑ 2013 Mărdărescu et al; licensee BioMed Central Ltd. This is an Open Access article distributed under the terms of the Creative Commons Attribution License (http://creativecommons.org/licenses/by/2.0), which permits unrestricted use, distribution, and reproduction in any medium, provided the original work is properly cited. 